\title{
Dual Stator Winding Induction Generator (DSWIG) used as base source in hybrid power systems when lost the power
}

\author{
MARCEL TOPOR ${ }^{1}$, SORIN IOAN DEACONU ${ }^{1}$ Senior Member IEEE, GABRIEL NICOLAE \\ POPA Senior Member IEEE, FEIFEI BU Senior Member IEEE \\ ${ }^{1}$ Department of Electrical Engineering and Industrial Informatics \\ ${ }^{2}$ Department of Electrical Engineering \\ ${ }^{1}$ Politehnica University Timisoara \\ ${ }^{2}$ Nanjing University of Aeronautics and Astronautics \\ ${ }^{1}$ Hunedoara, no. 5 Revolutiei Street \\ ${ }^{2}$ Nanjing, China \\ ${ }^{1}$ ROMANIA \\ ${ }^{2}$ CHINA
}

\begin{abstract}
In this paper we propose a completely new solution in which the diesel generator is replaced by a gas turbine that drives a dual stator winding induction generator (DSWIG), which can operate in a wide range of speeds, thus enabling maximization the efficiency of the gas turbine utilization according to the power requirements of the critical supply network.
\end{abstract}

Key-Words: - induction generator, dual stator winding, gas turbine, emergency power supply, hybrid system

Received: January 16, 2020. Revised: August 31, 2020. Re-revised: September 8 , 2020.

Accepted: September 15, 2020. Published: September 26, 2020.

\section{Introduction}

Electricity supply to critical consumers has been and is a major concern in the field of low-voltage electrical networks. Critical consumers include: hospitals, data storage centers, communications equipment, military equipment, hotels, showrooms, restaurants, elderly homes, children homes, office buildings, malls, etc.

The classic solution (with diesel generators) is overtaken at this time, considering the current pollution and noise requirements. In this paper we propose a completely new solution in which the diesel generator is replaced by a $g$ as turbine that drives a dual stator winding induction generator (DSWIG), which can operate in a wide range of speeds, thus enabling maximization the efficiency of the gas turbine utilization according to the power requirements of the critical supply network.

\section{Conventional emergency power solutions for critical consumer's example}

An extremely important feature of modern power supply is the redundancy and uptime of the energy provision service. For new or critical consumers without redundant systems or components somewhere it is very common to have a redundant system for energy supply. Usual examples in the IT or military include multiple redundant power supplies. The set-up typically includes redundant uninterruptable power supply (UPS) systems, generators and even redundant utility feeds to support a reliable facility. However there some potential singlepoint-of-failure flaws hidden this rather classical electrical design [1-3].

One of the most concerning situations is related by the use of the Automatic Transfer Switch if not carefully designed the ATS becomes single point of failure in the system. Automatic transfer switch can become a blockage since the reliability of the protection depends on the ATSes performance. Especially designs with backup generators present a vulnerability given by these automatic transfer switches (ATSes), which sense the power failure, start the emergency generators and switch the load to them when the generator power is stabilized. Despite all the other redundancies taken in consideration from the design stage, a f ailure of either ATS or its circuit breakers will bring down the entire power supply system. For example, if the ATS feeding the switchboard fails, the consumer will immediately shut down when the alternative power source (batteries) are depleted.

For a reliable solution the use of diesel gensets need to be equipped with two starters (electrical, air, or 
hydraulic starting system), but in this way the genset is expensive and will require frequent and costly maintenance. Another drawback is the fact that diesel gensets are source of pollution and noise and their use is limited in some environments. An alternate design may include a battery bank with long backup time. This solution requires space considering replacement and maintenance, battery banks are one of the most expensive equipment's in microgrid systems. To avoid these drawbacks a reliable, less frequent and cost effective maintenance, a relative environmental friendly power system is established by using hybrid power micro grid architecture. There is a large initiative to use this solution. The main effect in using a micro grid is the fact that this way we can eliminate the dependence on diesel gensets. While some micro grids were typically powered by diesel, these micro grid architecture proposed in this paper can be powered by fuel cells, solar, wind, combined heat and power, and batteries instead of or in addition to diesel [4-7].

Renewable energy infusion in the power supply provides the benefit of reliability because it will not run out, as fossil fuels might, during an emergency. The micro grid allows a high penetration of renewable thus a sen sible reduction on the energy costs. Mostly in natural disaster situations the hybrid micro grid is capable to sustain critical consumers like a hospital, a military base, a flood pumping system or an emergency command center.

\section{Architecture of the proposed hybrid micro grid with renewable power sources for critical consumers}

The proposed micro grid architecture is presented in figure 1. A key element of the proposed micro grid is a dual stator winding induction generator DSWIG. The DSWIG is basically an induction generator equipped with two independent stator windings. What it is interesting on this type of generator is the fact that the machine is multiport generator capable to provide energy on two separate windings. Its stator windings are separated from one set into two sets: one is called as the control winding $(\mathrm{CW})$, which provides the variable excitation reactive power for the generator; the other is referred to as the power winding (PW), which outputs the active power to the loads.

The two sets of stator windings have the same pole pairs, and there is no electrical connection but electromagnetic link between them. Since the converter is not connected directly with the loads, the high frequency currents induced by the power switches have little effect on the loads on the PW s ide. And the excitation reactive power is independent and can be easily regulated from the $\mathrm{CW}$ side through the static excitation converter. The DSWIG is an advancement the single power supply pattern like AC or DC power supply cannot meet the new requirements of the diversity of electric power patterns as both AC and DC loads may exist in the system. In this way the generator can be operated in a hybrid generating system which is a good s olution to the problem of redundancy. One typical topology is presented in [8], [9], as shown in figure 1. In this system, the PW outputs the DC power directly, while the $\mathrm{CW}$ provides not only variable reactive power for the DSWIG but also DC power via the DC bus to the loads.

A few seconds after the main (and possibly secondary) power network are lost, the gas micro turbine is switched on and the DSWIG generates power, with help of a battery of capacitors that permanently magnetizes the machine magnetic core, placed in the stator auxiliary winding. To optimize the operation, it is proposed to use a combination of several power sources the photovoltaic source, which can be developed over time and which contributes to reducing network consumption and energy costs. It is also proposed to use as power sources or storage elements, electric vehicles (EVs) or hybrid plug-in vehicles, parked in the critical consumer area, to be connected to the grid and to be able to operate after the vehicle to grid (V2G) or grid to vehicle $(\mathrm{G} 2 \mathrm{~V})$ technology.

For the integration in the micro grid we have considered the micro turbine powered DSWIG. The reason we have used this solution is that the micro turbines is that we need a fast reliable primary energy source capable to provide are very small size gas turbines with sizes as small as $1 \mathrm{~kW}$ but it can be scaled up to $500 \mathrm{~kW}$ which meets our requirement. These small turbines usually have a single stage compressor and a single stage turbine, with the generator mounted on gear stage. Rotational speed is high, usually greater than $40,000 \mathrm{rev} /$ minutes and they require power electronics are used to fit the output frequency to the grid. Their efficiency is not low since many of the more efficient micro turbines recently build uses a heat recuperator to improve performance. The advantage is that these micro turbines can operate on any fuel gas, diesel LPG, and their emissions are lower than the corresponding diesel gensets [10].

The model presented in this paper is based on the low speed dynamics of the micro turbine system, suitable for power management of genset combined with other types of distributed generation (DG) systems. For micro grid simulation we can assume that the system is operating under normal operating conditions by neglecting fast dynamics of the micro turbine. The main functions of the micro turbine are: speed control block for part load conditions, 
temperature control function for upper output power limit, and acceleration control to prevent over speeding. The output of the control function blocks are all inputs to a least value gate (LVG), whose output is the lowest of the three inputs and controls in the amount of fuel to the compressor-turbine as shown in Figure 1.

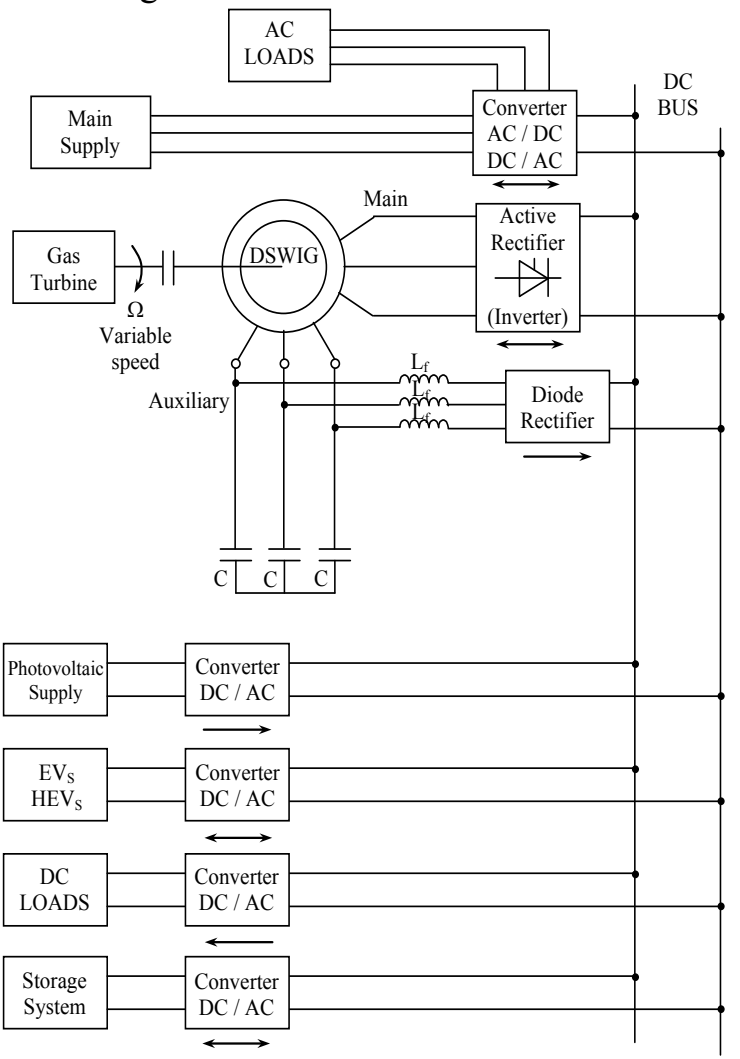

Fig. 1 Block scheme of proposed system.

$$
\begin{gathered}
V_{c e}=\frac{K\left(T_{1} s+1\right)}{T_{2} s+Z}\left(\omega_{\text {ref }}-\omega_{\text {meas }}\right), \\
W_{f \text { speed }}=\left(V_{c e} \cdot N \cdot K_{3} \cdot e^{-s T}+K_{6}\right) \underbrace{\frac{K_{v}}{T_{v} s+c}}_{\begin{array}{c}
\text { valve } \\
\text { positioner }
\end{array}} \frac{K_{f}}{\text { Fuel system }_{f} s+c} \\
W_{f L V G}=\left\{\begin{array}{l}
W_{f \max } \text { if } W_{f} \leq W_{f \min } \\
W_{f} \text { if } W_{f} \geq W_{f \max } \\
W_{f \max } \text { if } W_{f} \geq W_{f \max }
\end{array}\right.
\end{gathered}
$$

The acceleration control of the micro turbine rotation motion can be divided into 2 periods - a concave period followed by a convex period [11].

$$
\text { Concave }\left\{\begin{array}{l}
v(t)=v_{o}+j_{m} \frac{t^{2}}{2} \\
a(t)=j_{m} t
\end{array}\right.
$$

convex

$$
\left\{\begin{array}{l}
v(t)=v_{h}+a_{s} t-j_{m} \frac{t^{2}}{2} \\
a(t)=a_{s}-j_{m} t
\end{array},\right.
$$

where $j_{m}$ is the jerk set for the profile (near the maximum allowed for the micro turbine ), and $a_{s}$ is the maximum acceleration encountered at the Scurve inflection point

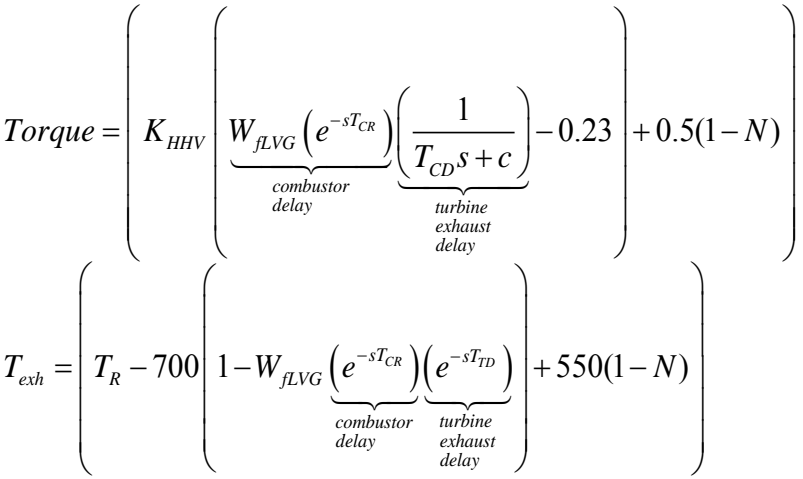

$$
\begin{aligned}
& W_{\text {temp }}=(T_{\text {ref }}-T_{\text {exh }} \cdot \underbrace{\left(K_{4}+\frac{K_{5}}{T_{3} S+1}\right)}_{\text {radiation shield }} \underbrace{\left(\frac{1}{T_{4} s+1}\right)}_{\text {Thermocouple }})\left(\frac{T_{5} S+1}{T_{t} S}\right) \\
& W_{\text {tempLVG }}=\left\{\begin{array}{l}
W_{\text {temp } \max } \text { if } W_{f} \leq W_{f \min } \\
W_{\text {temp }} \text { if } W_{f \min }<W_{f}<W_{f \max } \\
W_{\text {temp } \max } \text { if } W_{f} \geq W_{f \max }
\end{array}\right. \\
& W_{\text {fout }}=\min \left(W_{\text {fFlow }}, W_{\text {fTemp }}, W_{\text {fspeed }}\right)
\end{aligned}
$$

\section{Battery modelling}

The batteries are often considered as being the weakest point of a hybrid system, in terms of cost, life time and reliability. HRES is less suitable to charge batteries than the main grid because the battery elements are subjected to variable currents (irregular charge cycles). The battery model exploits equation to calculate the SOC in charge/discharge conditions. The prediction of state of charge on the battery is calculated using equations [12]:

$$
S O C=S O C_{0}+\int_{0}^{t} \frac{I_{b a t}}{C_{b a t}},
$$

where $C_{b a t}, S O C_{0}$ and $\mathrm{I}_{\text {bat }}$ represent capacity of battery $(\mathrm{Ah})$, and initial state of charge and battery current (A).

The hybrid generation system includes a special control system for the battery management called BMS. In the proposed system the BMS is equipped with MPPT and boost converters in order boosts a variable $\mathrm{DC}$ voltage output of $\mathrm{PV}$ to the required value of the dc grid and adapt it to DC voltage to the reference DC bus (48 VDC). The maximum available power from a PV hybrid system can be extracted when MPPT control applied. For modern BMS it is almost generalized solution to use a Perturb-and-observe ( $\mathrm{P} \& \mathrm{O})$ strategy to produce available duty cycle of PWM signal to control the 
boost converter. The MPPT system identifies the maximum available power from the PV generation system, and then compares the calculated power with the actual drawn power for generating the switching signals of the DC-DC boost converter of the MPPT system. Another DC-DC boost converter cascaded after the MPPT converter which order to maintain the voltage level of the DC bus at the value of 48 Volt to ensure continuous power supply to the DC load.

The state equation that characterizes the electrical modelling of the DC-DC boost converter is given here, where $\mathrm{S}$ is the switch state that takes the value 1 or $0, V_{i}$ is the input voltage to the $\mathrm{dc}-\mathrm{dc}$ converter (output from each energy source) and Vo is the dc link output voltage.

$$
\left[\begin{array}{c}
\frac{d V_{0}}{d t} \\
\frac{d I_{l}}{d t}
\end{array}\right]=\left[\begin{array}{cc}
\frac{1-S}{C} & \frac{-1}{R C} \\
0 & \frac{1-S}{L}
\end{array}\right]\left[\begin{array}{l}
V_{0} \\
I_{l}
\end{array}\right]+\left[\begin{array}{l}
0 \\
\frac{1}{L}
\end{array}\right] \cdot V_{i}
$$

The values of the capacitor and inductor are calculated as [13]:

$$
\begin{aligned}
L_{\text {min }} & =\frac{\left(D(1-D)^{2} R\right)}{2 f} \\
C & =\frac{D}{R\left(\left(\Delta V_{0}\right) / V_{0}\right) f}
\end{aligned}
$$

Taking the case of PVWT systems, the terminal voltage $V_{0}$ is controlled by the voltage error signal. Where the PV voltage and current are sensed to determine the reference voltage at which MPPT occurs, the error signal, which is the difference between the reference voltage, and the actual voltage of the $\mathrm{PV}$ is fed to the voltage controller to command the duty cycle of the PV boost converter.

For the micro turbine the error signal is the difference between the reference of rectified voltage of the DSWIG and measured rectified voltage. This error signal is fed to the voltage controller, which controls the duty cycle of the boost converter.

$$
P_{\text {batt }}=\Delta P=P_{s}-P_{P V}-P_{M T}
$$

The sum of electricity generated at both generators photovoltaic and micro turbine (PVMT) is designed to meet the required DC load demand since the output power of PV generators vary with irradiation. The battery output power is controlled based on the difference power command DP, which is the load power (command value) Ps minus the summation of the power generated from the PVMT. The battery terminal voltage is calculated according the desired battery power using a look-up table, the input of this look-up table is the difference power command DP, which means the battery reference or required power.

\section{DSWIG control strategy}

The output voltage and control variable are described using the instantaneous power theory [9]. For the generator control we use field oriented of the control-winding flux $\psi_{c 1}$ direction, the instantaneous active $p_{c 1}$ and reactive power $q_{c 1}$ are

$$
\left\{\begin{array}{l}
p_{c 1}=\omega_{1} \psi_{c 1} i_{c q 1} \\
q_{c 1}=\omega_{1} \psi_{c 1} i_{c d 1}
\end{array},\right.
$$

where $i_{c d 1}, i_{c q 1}$ are the $d-q$ of control-winding current $\left(\mathrm{i}_{\mathrm{c} 1}\right) . \Psi_{\mathrm{c} 1}$ is the amplitude of $\psi_{\mathrm{c} 1}$. The torque and flux are [14]:

$$
\left\{\begin{array}{l}
T_{e c 1}=n_{p} \psi_{c 1} i_{c q 1} \\
\psi_{c 1}=L_{c} i_{c d 1}+\psi_{p c M}+\psi_{r c M}
\end{array}\right.
$$

where $\mathrm{n}_{\mathrm{p}}$ is the pole pairs, $L_{c}$ is the inductance, $\psi_{p c M}$ and $\psi_{\text {rcm }}$ are the mutual flux of power winding to control winding and control winding to rotor, respectively. Output voltages are:

$$
\left\{\begin{array}{l}
u_{p D C}=2.65 \cdot u_{p} \\
u_{c D C}=\left(\omega_{1}\left|T_{e c 1}\right| R_{E}\right)^{\frac{1}{2}},
\end{array}\right.
$$

where $u_{p}$ is the root mean square (rms) of powerwinding phase voltage. $R_{E}$ is the equivalent resistance. Therefore, the output voltages can be regulated by the control of fundamental current loop.

\section{Grid Side Power Control}

The inverter 3 in Figure 2 is connected between the DC bus and the electrical network through RL filter. This inverter has two functions: to keep the DC bus voltage constant, irrespective of the amplitude and direction of the power flow and to maintain a unit power factor at the point of connection with the electrical network [11]. The mathematical modeling of the studied DC link system is as follows

$$
\frac{\mathrm{dU}}{\mathrm{dt}}=\frac{1}{C}\left(i_{d c}-i_{m}\right)
$$

where:

$$
i_{d c}=i_{d 1}+i_{d 2}
$$

Expressions of grid reference currents can be characterized by:

$$
\left\{\begin{array}{l}
i_{d g}=\frac{1}{\left(R_{t}+L_{t} s\right)}\left(v_{d-i n v}-v_{d g}-L_{t} \omega_{s} i_{q g}\right) \\
i_{d g}=\frac{1}{\left(R_{t}+L_{t} s\right)}\left(v_{q-i n v}-v_{q g}-L_{t} \omega_{s} i_{d g}\right)
\end{array}\right.
$$

The grid active and reactive powers are defined by 


$$
\left\{\begin{array}{l}
P_{s}=v_{d g} i_{d g}+v_{q g} i_{q g} \\
Q_{s}=v_{d g} i_{d g}-v_{q g} i_{q g}
\end{array}\right.
$$

The block diagram of the control loops for the currents axis $(\mathrm{d}, \mathrm{q})$ is described in figure 2. The correctors used are of the PI type. This block diagram includes the terms of decoupling and compensation in order to be able to independently control the currents circulating in the RL filter and the active and reactive powers exchanged between the CCR and the network.

\section{Grid control of active power}

In order to control the flow of active power, the value $P_{i j}$, of the real power flow between node $I$ and node $j$ is given by

$$
P_{i j}=\frac{1}{X_{i j}}\left(\theta_{i}-\theta_{j}\right)
$$

The total power flowing into bus (node) $I$ is denoted as $P_{i}$. This must equal the power generated by generator $I$ minus the power absorbed by the local load on the bus. This power, $P_{i}$, therefore must equal the sum of the power flowing away from bus $I$ on all transmission lines. This means that

$$
P_{i j}=\sum_{j \in N(\mathrm{i})} P_{i j}=\sum_{j \in N(\mathrm{i})}\left(\theta_{i}-\theta_{j}\right) \frac{1}{X_{i j}}
$$

which can be expressed in matrix form as

$$
P_{i j}=B \theta
$$

where $P=\left[P_{1}, \ldots, P_{N}\right], q=\left[q_{1}, \ldots, q_{N}\right]$, and $B$ is defined as

$$
B=\left\{\begin{array}{ll}
\sum_{j \in N(\mathrm{i})} \frac{1}{x_{i j}} & \text { if } i=j \\
\frac{1}{X_{i j}} & \text { if } \mathrm{e}_{i j} \in E \\
0 & \text { if } \mathrm{e}_{i j} \notin E
\end{array} .\right.
$$

The $C_{i}(P) \in R$ is a function representing the cost incurred in running generator $I$ at power level $\mathrm{P}$. The equation formula for a general optimal power flow problem it comes as

$$
\left\{\begin{array}{c}
\text { minimize } \sum_{i=1}^{N} C_{i}\left(P_{G i}\right) \\
\text { considering } P_{G 1}, \ldots P_{G N} \\
\text { with the constraints } B \theta=P_{G}-P_{L} \\
P_{\mathrm{G} \min } \leq P_{G} \leq P_{G \text { max }} \\
P_{\min } \leq A \theta \leq P_{\max }
\end{array}\right.
$$

where $P_{G}$ is the vector of generated active powers for all generators (micro turbine and solar in this case and $\mathrm{P}_{\mathrm{L}}$ is the vector of total local loads for all buses. The matrices $A$ and $B$ were defined above. The vector $P_{\mathrm{G} \min }$ and $P_{\mathrm{G} \max }$ represent the lower and upper limits of the DSWIG generator power. These are the generation constraints. The other vectors, $P_{\min }$ and $P_{\max }$, are lower and upper limits on the power flowing through the distribution lines. The objective function $\sum_{i=1}^{N} C_{i}\left(P_{G i}\right)$ represents the total generation cost of all sources. The grid optimum control problem has the objective to minimize this overall cost by selecting generating powers (micro turbine DSWIG battery and solar that satisfy the major three constraints imposed to the micro grid. The first constraint is a power balance relation. The second constraint requires that the selected power levels stay within the limits specified by $P_{\mathrm{G} \min }$ and $P_{\mathrm{G} m a x}$. The third constraint requires that the power flowing over the distribution lines stay within the specified bounds, $P_{\min }$ and $P_{\max }$.

\section{Simulation of the proposed micro grid}

In order to simulate the behavior, the micro grid the corresponding models of the key components need to be presented. The corresponding equations are modeled in MATLAB Simulink model shown in figure 


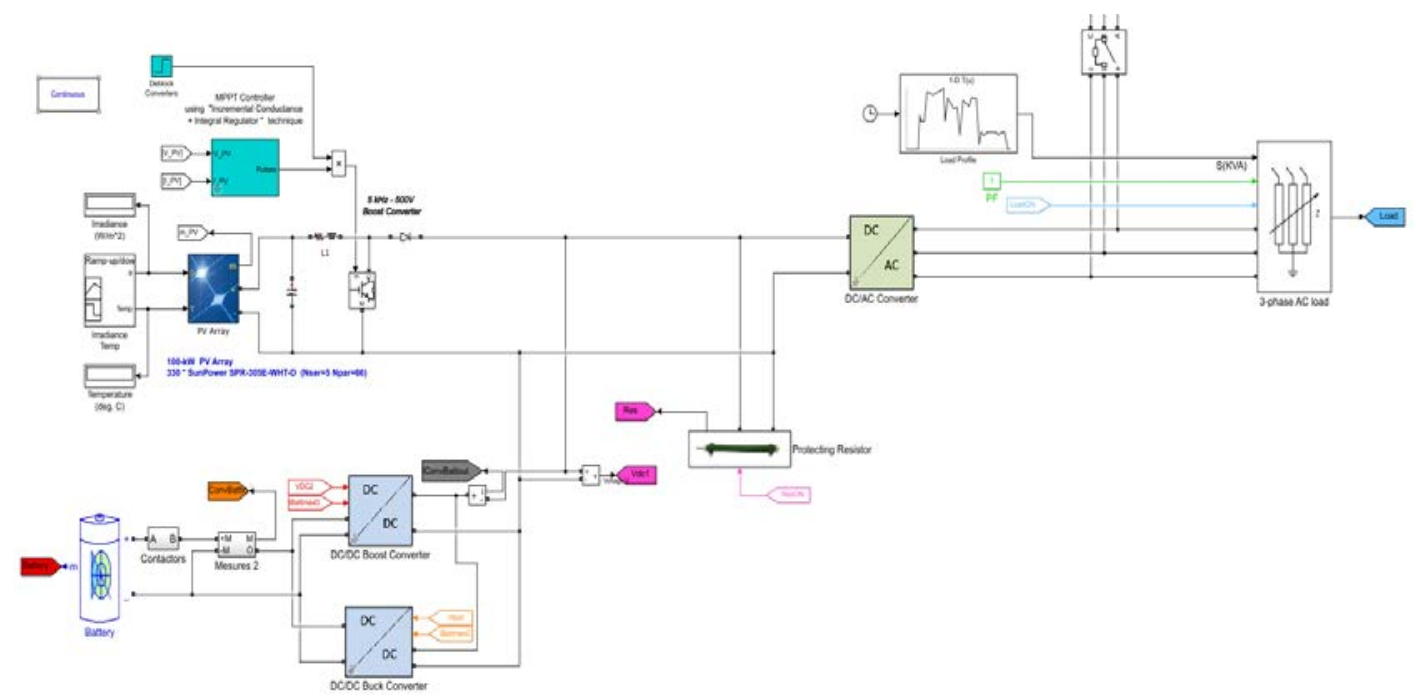

Figure 2. Hybrid Micro grid Simulink Model

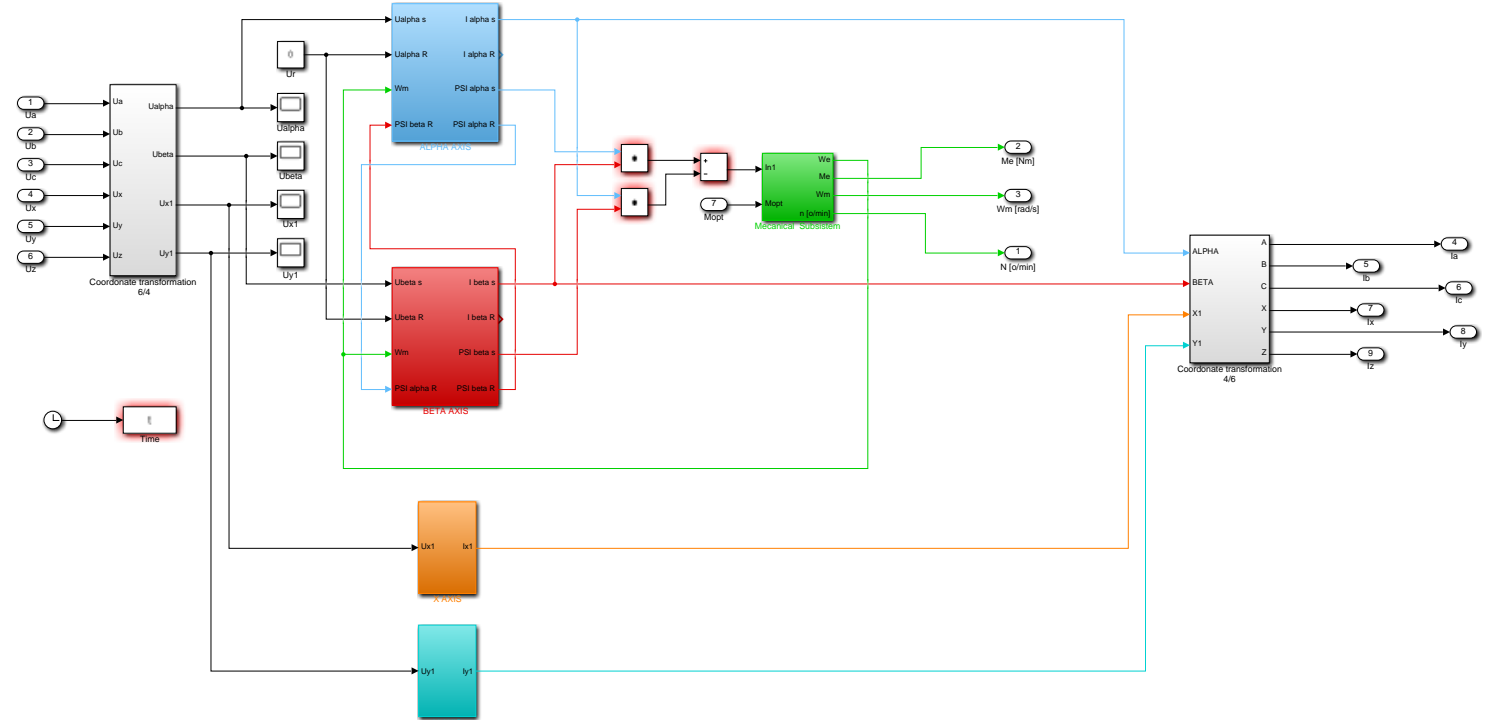

Figure 3. DSWIG model

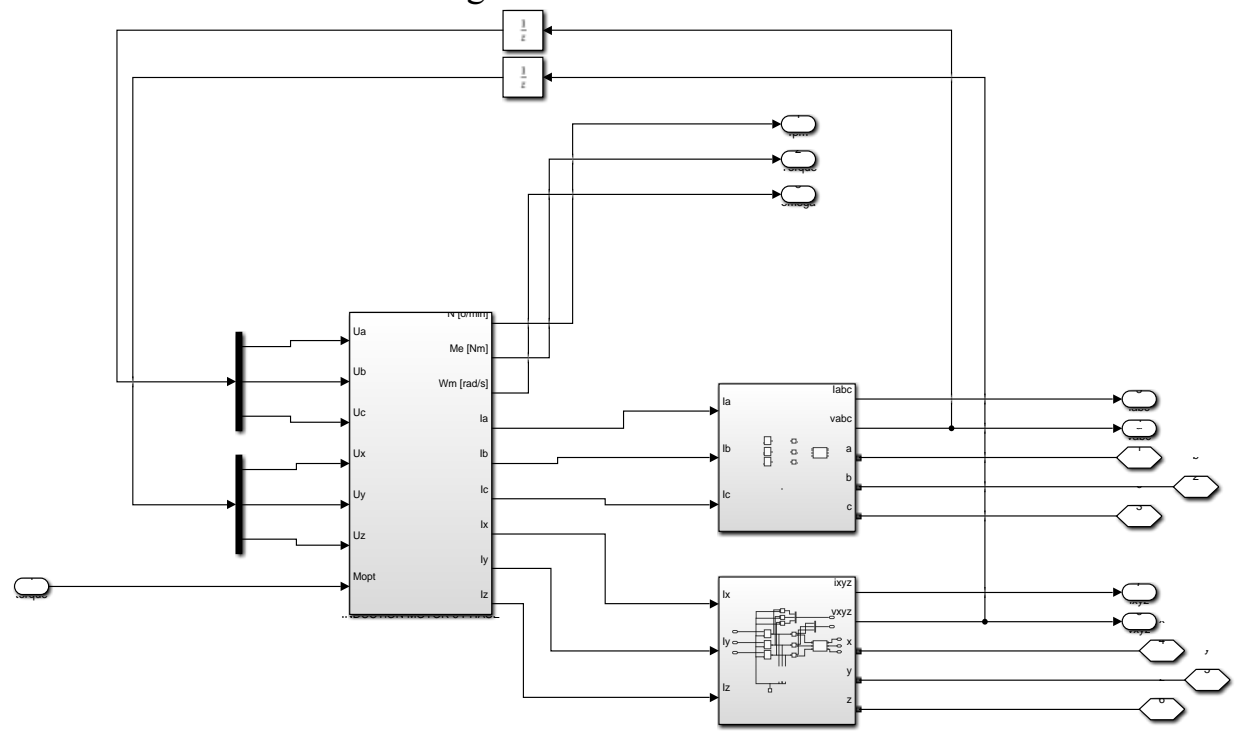

Figure 4. Microturbine DSWIG Genset configuration 


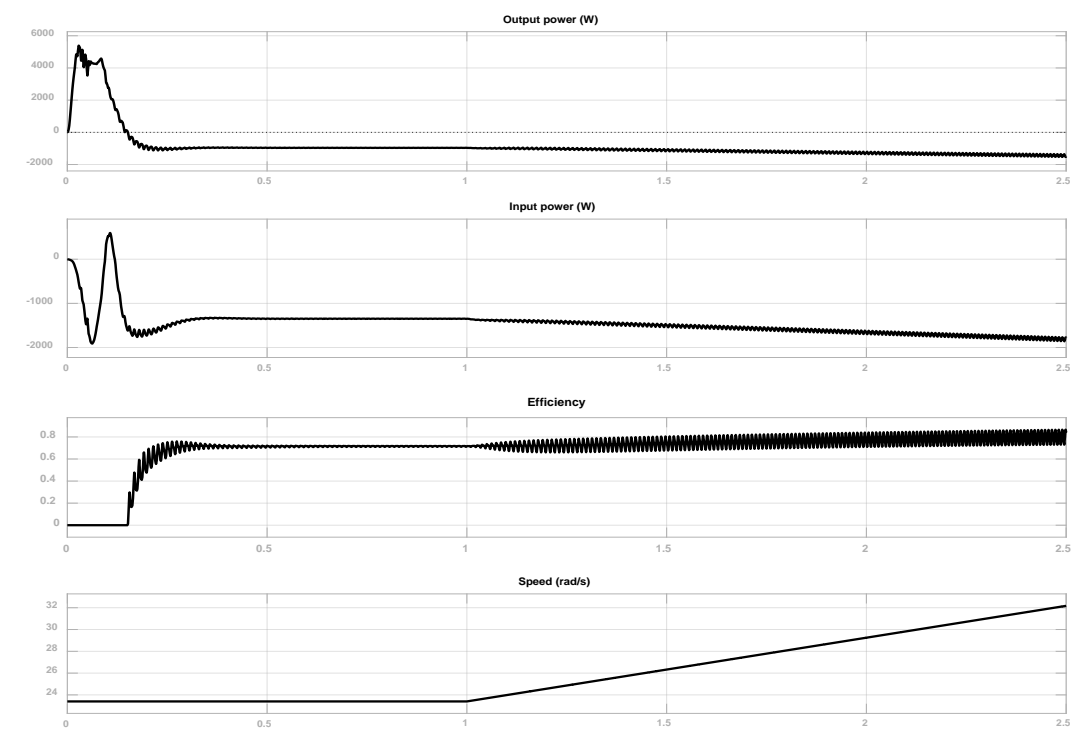

Figure 5. Figure with short caption (caption centered)

\section{Conclusions}

In this paper we have presented a hybrid micro grid system build on the basis of a new micro turbine dual stator induction generator connected to DC bus supplying a number DC loads. After, we have proposed a detailing diagram showing the flow direction of the electrical power, and showing also the optimization module MPPT which is using $\mathrm{P}$ $\& O$ algorithm and we had justifying the use of the DC-DC boost converter and its control mode by PWM. Then we have used a detailed mathematical model of each RES. The mathematical models were implemented models into a large Simulink model system that allows us the study of the corresponding characteristic and their diagram model. The model simulation using Simulink model is used for the tuning and control of the electrical power at 500 volt DC. The hybrid micro grid integrating solar and micro turbine DSWIG scheme proposed in this paper uses an inverter with apparent power lower than the corresponding generator power. The expected ratio between the inverter power and the generator power is $50 \%$ in the case of DSWIG.

\section{References:}

[1] A. Hussain, V. Bui and H. Kim, Robust Optimal Operation of AC/DC Hybrid Microgrids Under Market Price Uncertainties, in IEEE Access, vol. 6, pp. 2654-2667, 2018.

[2] A. Kumar, A. R. Singh, Y. Deng, X. He, P. Kumar and R. C. Bansal, A Novel Methodological Framework for the Design of Sustainable Rural Microgrid for Developing Nations, in IEEE Access, vol. 6, pp. 2492524951, 2018.
[3] M. Starke, A. Herron, D. King and Y. Xue, Implementation of a Publish-Subscribe Protocol in Microgrid Islanding and Resynchronization With Self-Discovery, in IEEE Transactions on Smart Grid, vol. 10, no. 1, pp. 361-370, Jan. 2019.

[4] P. Wu, W. Huang and N. Tai, Advanced design of microgrid interface for multiple microgrids based on MMC and energy storage unit, in The Journal of Engineering, vol. 2017, no. 13, pp. 2231-2235, 2017.

[5] I. U. Nutkani, L. M eegahapola, L. P. C. Andrew and F. Blaabjerg, Autonomous power management for interlinked AC-DC microgrids, in CSEE Journal of Power and Energy Systems, vol. 4, no. 1, pp. 11-18, March 2018.

[6] T. L. Nguyen, Q. Tran, R. Caire, Y. Besanger, T. T. Hoang and V. H. Nguyen, FMI compliant approach to investigate the impact of communication to islanded microgrid secondary control, 2017 IEEE Innovative Smart Grid Technologies - Asia (ISGT-Asia), Auckland, 2017, pp. 1-6.

[7] T. Ma, J. Wu and X. Niu, Reliability assessment indices and method for urban microgrid, in CIRED - Open Access Proceedings Journal, vol. 2017, no. 1, pp. 837840, 102017.

[8] M. Batool, S. M. Islam and F. Shahnia, Power transaction management amongst coupled microgrids in remote areas, 2017 IEEE Innovative Smart Grid Technologies - Asia (ISGT-Asia), Auckland, 2017, pp. 1-6.

[9] Y. Guo, S. Li, C. Li and H. Peng, Short-Term Reliability Assessment for Islanded Microgrid Based on Time-Varying Probability Ordered 
Tree Screening Algorithm, in IEEE Access, vol. 7, pp. 37324-37333, 2019.

[10] M. Omar and G. Scarcella, Unbalanced and reactive power compensation for grid friendly microgrids, 3rd Renewable Power Generation Conference (RPG 2014), Naples, 2014, pp. 16.

[11] G. K. Venayagamoorthy, R. K. Sharma, P. K. Gautam and A. Ahmadi, Dynamic Energy Management System for a Smart Microgrid, in IEEE Transactions on Neural Networks and Learning Systems, vol. 27, no. 8, pp. 16431656, Aug. 2016.

[12] L. Jia, Y. Zhu, S. Du and Y. Wang, Analysis of the transition between multiple operational modes for hybrid AC/DC microgrids, in CSEE Journal of Power and Energy Systems, vol. 4, no. 1, pp. 49-57, March 2018.

[13] G. Haines, N. Ertugrul, G. Bell and M. Jansen, Microgrid system and component evaluation: Mobile test platform with battery storage, 2017 IEEE Innovative Smart Grid Technologies - Asia (ISGT-Asia), Auckland, 2017, pp. 1-5.

[14] Z. Yi, X. Zhao, D. Shi, J. Duan, Y. Xiang and Z. Wang, Accurate Power Sharing and Synthetic Inertia Control for DC Building Microgrids With Guaranteed Performance, in IEEE Access, vol. 7, pp. 63698-63708, 2019.

\section{Creative Commons Attribution License 4.0} (Attribution 4.0 International, CC BY 4.0)

This article is published under the terms of the Creative Commons Attribution License 4.0

https://creativecommons.org/licenses/by/4.0/deed.en_US 